\title{
A finite element method for transient analysis of concurrent large deformation and mass transport in gels
}

\section{Citation}

Zhang, Jiaping, Xuanhe Zhao, Zhigang Suo, and Hanqing Jiang. 2009. "A Finite Element Method for Transient Analysis of Concurrent Large Deformation and Mass Transport in Gels." Journal of Applied Physics 105 (9): 93522. https://doi.org/10.1063/1.3106628.

\section{Permanent link}

http://nrs.harvard.edu/urn-3:HUL.InstRepos:41461222

\section{Terms of Use}

This article was downloaded from Harvard University's DASH repository, and is made available under the terms and conditions applicable to Other Posted Material, as set forth at http:// nrs.harvard.edu/urn-3:HUL.InstRepos:dash.current.terms-of-use\#LAA

\section{Share Your Story}

The Harvard community has made this article openly available. Please share how this access benefits you. Submit a story.

Accessibility 


\title{
A finite element method for transient analysis of concurrent large deformation and mass transport in gels
}

\author{
Jiaping Zhang, ${ }^{1}$ Xuanhe Zhao, ${ }^{2}$ Zhigang Suo, ${ }^{2}$ and Hanqing Jiang ${ }^{1, a)}$ \\ ${ }^{1}$ Department of Mechanical and Aerospace Engineering, Arizona State University, Tempe, Arizona 85287, \\ USA \\ ${ }^{2}$ School of Engineering and Applied Sciences, Harvard University, Cambridge, Massachusetts 02138, USA
}

(Received 3 December 2008; accepted 23 February 2009; published online 8 May 2009; publisher error corrected 21 May 2009)

\begin{abstract}
A gel is an aggregate of polymers and solvent molecules. The polymers crosslink into a three-dimensional network by strong chemical bonds and enable the gel to retain its shape after a large deformation. The solvent molecules, however, interact among themselves and with the network by weak physical bonds and enable the gel to be a conduit of mass transport. The time-dependent concurrent process of large deformation and mass transport is studied by developing a finite element method. We combine the kinematics of large deformation, the conservation of the solvent molecules, the conditions of local equilibrium, and the kinetics of migration to evolve simultaneously two fields: the displacement of the network and the chemical potential of the solvent. The finite element method is demonstrated by analyzing several phenomena, such as swelling, draining and buckling. This work builds a platform to study diverse phenomena in gels with spatial and temporal complexity. (C) 2009 American Institute of Physics. [DOI: 10.1063/1.3106628]
\end{abstract}

\section{INTRODUCTION}

Long-chain polymers may crosslink by strong chemical bonds into a three-dimensional network. The resulting material, an elastomer, is capable of large and reversible deformation. The elastomer may imbibe a large quantity of solvents, aggregating into a gel (Fig. 1). The solvent molecules in the gel interact by weak physical bonds and can migrate. The dual attributes of a solid and a liquid make the gel a material of choice in nature and in engineering. For example, gels constitute many tissues of animals and plants. The solid attribute enables the tissues to retain shapes, while the fluid attribute enables the tissues to transport nutrients and wastes. Gels are also synthesized for diverse applications, including food processing, ${ }^{1}$ drug delivery, ${ }^{2-5}$ tissue engineering, ${ }^{6,7}$ actuators in miniaturized devices, ${ }^{8-10}$ and packers in oil wells. ${ }^{11}$

Many processes in gels involve concurrent deformation and migration. For example, a drug loaded in a gel can migrate out in response to a change in the physiological conditions (i.e., the temperature, the level of $p \mathrm{H}$, or the concentration of an enzyme). The rate of the release may be modulated by the deformation of the gel (e.g., Ref. 12). As another example, patterns of crease often appear on the surface of a swelling gel (e.g., Refs 13 and 14), along with many other forms of buckling. ${ }^{15-18}$ Furthermore, swelling may induce stress localization in gels, which leads to cavitation and delamination. ${ }^{19}$ Hydrogels with submillimeter size have been extensively used as valves in microfluidics due to the short swelling time and large deformation. ${ }^{8}$

This paper studies the concurrent deformation and migration in the gel by a finite element method. Our method builds upon a theory dating back to Gibbs (1878), who formulated a thermodynamic theory of mobile molecules in an

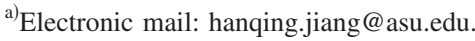

elastic solid. Biot (1941) combined the thermodynamic theory and Darcy's law for mass transport in a porous medium. Both Gibbs and Biot used phenomenological freeenergy functions, and their works were not specific for the polymeric gel. Using statistical mechanics, Flory and Rehner $^{20}$ developed a free-energy function for the gel, including the effects of the entropy of stretching the network, the entropy of mixing the network polymers and the solvent molecules, and the enthalpy of mixing. Reviews of subsequent contributions to the theory of polymeric gels are found, among others, in Refs. 15 and 21-26. Another theory that is able to study the deformation and migration is the mixture theory, ${ }^{27}$ in which the fluid and solid follow different field equations.

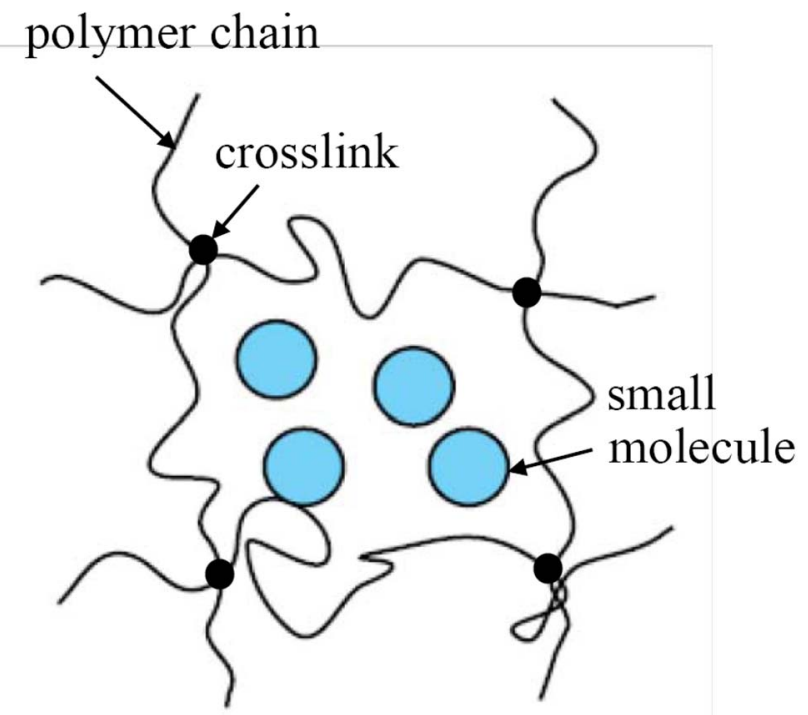

FIG. 1. (Color online) A schematic of structure of a gel. 
There have been several previous efforts to develop finite element methods for gels. Westbrook and $\mathrm{Qi}^{28}$ and Hong et $a l^{29}$ have developed finite element methods for gels in a state of equilibrium. Suematsu et al. ${ }^{30}$ conducted the threedimensional explicit finite element analysis to study the pattern formation of swelling gels by introducing a friction constant between the polymeric chains and solvents. ${ }^{15}$ As pointed out by Suematsu et al. ${ }^{30}$ this method is not suited for larger systems over longer time intervals. Dolbow et al. ${ }^{22}$ used a hybrid eXtended-Finite-Element/Level-Set method to study the swelling of gels. Li et al..$^{24}$ used an explicit method to alternately solve the coupled problems for gels, namely, the deformation of gels is solved after the convergent results for mass transport is obtained. Birgersson et al. ${ }^{31}$ conducted transient analysis of temperature-sensitive two-dimensional gels by using finite element solver, COMSOL MULTIPHYSICS.

Given various theories and numerical methods, as well as a large number of phenomena and applications, ample room exists for more computational work to connect principles of mechanics, thermodynamics, and kinetics to experiments and to molecular models. In particular, we will develop a finite element method using the free-energy function of Flory and Rhener (1943) and the kinetic model proposed by Hong et al. ${ }^{26}$ The implicit method for time discretization is used, and transport and deformation are solved concurrently. We will implement the method in ABAQUS via a userdefined element (UEL).

The plan of the paper is as follows. Section II outlines the theory in a form suitable for the finite element method. The gel undergoes an irreversible thermodynamic process that simultaneously evolves two fields: the displacement of the network and the chemical potential of the solvent. Section III describes the material model: the free-energy function and the mobility tensor. Section IV describes aspects of finite element implementation. Section V demonstrates the method by analyzing several time-dependent processes for three-dimensional gels, including swelling, draining, and buckling.

\section{THEORY OF CONCURRENT DEFORMATION AND MIGRATION IN A GEL}

This section summarizes the theory of concurrent deformation and migration, describing in turn the kinematics of the network, the conservation of the solvent, the conditions of local equilibrium, and the kinetics of migration. The theory is essentially that of Gibbs (1978) and Biot (1941), and the notation follows that of Hong et al. ${ }^{26,29}$

\section{A. Kinematics of the network}

We use a standard approach in continuum mechanics to describe the kinematics of the network. The gel moves in a three-dimensional space. Imagine that each differential element of the network is attached with a marker. Any configuration of the gel can serve as a reference configuration (Fig. 2 ). When the gel is in the reference configuration, the marker occupies in the space a place with coordinates $\mathbf{X}$, which we will use to label the marker. In the reference configuration,

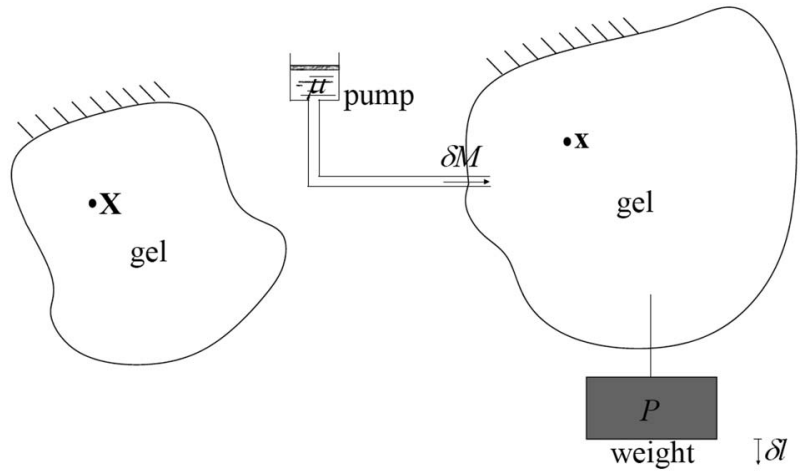

reference configuraiton

current configuraiton

FIG. 2. The reference and current configurations with an illustration of two ways doing work on a gel in the current configuration: a mechanical loading is applied by hanging a weight and a chemical loading is applied by using a pump to inject small molecules into the gel.

let $d V(\mathbf{X})$ be an element of volume, $d A(\mathbf{X})$ be an element of area, and $N_{K}(\mathbf{X})$ be the unit vector normal to the element of area.

At time $t$, the gel is in the current configuration, and the marker $\mathbf{X}$ moves in the space to a new place with coordinates $\mathbf{x}$. The functions $x_{i}(\mathbf{X}, t)$ specify the kinematics of the network. As usual, the deformation gradient of the network is defined as

$$
F_{i K}(\mathbf{X}, t)=\frac{\partial x_{i}(\mathbf{X}, t)}{\partial X_{K}} .
$$

We will use $\mathbf{F}$ to characterize the state of deformation of an element of the gel.

\section{B. Conservation of the solvent molecules}

We next use nominal quantities to describe the conservation of the solvent molecules. Let $C(\mathbf{X}, t)$ be the nominal concentration of the solvent in the gel in the current configuration, namely, $C(\mathbf{X}, t) d V(\mathbf{X})$ is the number of solvent molecules in the element of volume. Let $J_{K}(\mathbf{X}, t)$ be the nominal flux of the solvent in the gel, namely, $J_{K}(\mathbf{X}, t) N_{K}(\mathbf{X}) A(\mathbf{X})$ is the number of the solvent molecules per unit time migrating across the element of area. Imagine that the network is attached with a field of pumps, which inject the solvent into the gel. In the current configuration, the pumps inject $r(\mathbf{X}, t) d V(\mathbf{X})$ number of the solvent molecules into the element of volume per unit time, and $i(\mathbf{X}, t) d A(\mathbf{X})$ number of the solvent molecules into the element of area per unit time. We assume that no chemical reaction occurs, so that the number of the solvent molecules is conserved, namely,

$$
\frac{\partial C(\mathbf{X}, t)}{\partial t}+\frac{\partial J_{K}(\mathbf{X}, t)}{\partial X_{K}}=r(\mathbf{X}, t),
$$

in the volume of the gel, and

$$
J_{K}(\mathbf{X}, t) N_{K}(\mathbf{X})=-i(\mathbf{X}, t),
$$

on the part of the surface of the gel where the pumps inject solvent molecules. 


\section{Conditions of local equilibrium}

We now examine the conditions of local equilibrium. Elements of the gel in different locations may not be in equilibrium with each other, and this disequilibrium motivates the solvent to migrate. Each differential element of the gel, however, is taken to be locally in a state of equilibrium. That is, the migration of the solvent is such a slow process that the effect of inertia is negligible, the viscoelastic process in the element has enough time to relax, and the solvent in the element has enough time to equilibrate with the solvent in the pump attached to the element. Furthermore, the gel is assumed to be held at a constant temperature. We characterize the thermodynamic state of the differential element of the gel by the deformation gradient of the network $\mathbf{F}(\mathbf{X}, t)$, and the chemical potential of the solvent $\mu(\mathbf{X}, t)$. Let $\hat{W}(\mathbf{F}, \mu)$ be a free-energy density function of the gel, namely, $\hat{W}(\mathbf{F}, \mu) d V(\mathbf{X})$ is the free energy associated with the element of the gel. The conditions of local equilibrium require that the nominal concentration be given by

$$
C=-\frac{\partial \hat{W}(\mathbf{F}, \mu)}{\partial \mu},
$$

and the nominal stress be given by

$$
s_{i K}=\frac{\partial \hat{W}(\mathbf{F}, \mu)}{\partial F_{i K}} .
$$

When the free-energy density function $\hat{W}(\mathbf{F}, \mu)$ is prescribed for a gel, Eqs. (4) and (5) constitute the equations of state.

Imagine that the network is attached with a field of weights, which apply forces to the gel. In the current configuration, the weights apply a force $B_{i}(\mathbf{X}, t) d V(\mathbf{X})$ to the element of volume, namely, $B_{i}(\mathbf{X}, t)$ is the applied forces in the current configuration per unit volume of the reference configuration. Similarly, the weights apply a force $T_{i}(\mathbf{X}, t) d A(\mathbf{X})$ to the element of area, namely, $T_{i}(\mathbf{X}, t)$ is the applied forces in the current configuration per unit area of the reference configuration. The conditions of local equilibrium require that the inertia effect be negligible and that the viscoelastic process in the element be fully relaxed, so that

$$
\frac{\partial s_{i K}(\mathbf{X}, t)}{\partial X_{K}}+B_{i}(\mathbf{X}, t)=0,
$$

in the volume of the gel, and

$$
s_{i K}(\mathbf{X}, t) N_{K}(\mathbf{X})=T_{i}(\mathbf{X}, t),
$$

on the part of the surface of the gel where forces are applied.

\section{Kinetics of migration}

We will also use the nominal quantities to describe the kinetics of migration. The flux of the solvent is taken to be linear to the gradient of the chemical potential of the solvent

$$
J_{K}=-M_{K L}(\mathbf{F}, \mu) \frac{\partial \mu(\mathbf{X}, t)}{\partial X_{L}},
$$

where $M_{K L}$ is the mobility tensor. The mobility tensor is symmetric and positive definite, and in general depends on the thermodynamic state of the element, namely, on local values of the deformation gradient and the chemical potential.

The above theory evolves the configuration of the gel, namely, evolves concurrently the two fields $\mathbf{x}(\mathbf{X}, t)$ and $\mu(\mathbf{X}, t)$, once the following items are prescribed:

- the initial conditions $\mathbf{x}\left(\mathbf{X}, t_{0}\right)$ and $\mu\left(\mathbf{X}, t_{0}\right)$ at a particular time $t_{0}$,

- the applied force $B_{i}(\mathbf{X}, t)$ and the rate of injection $r(\mathbf{X}, t)$ inside the gel,

- either $i(\mathbf{X}, t)$ or $\mu(\mathbf{X}, t)$ on the surface of the gel,

- either $T_{i}(\mathbf{X}, t)$ or $\mathbf{x}(\mathbf{X}, t)$ on the surface of the gel, and

- the free-energy function $\hat{W}(\mathbf{F}, \mu)$ and the mobility tensor $M_{K L}(\mathbf{F}, \mu)$.

\section{MATERIAL MODEL}

Within the theory presented in the previous section, a material model is specified by the functions $\hat{W}(\mathbf{F}, \mu)$ and the mobility tensor $M_{K L}(\mathbf{F}, \mu)$. Following Hong et al. ${ }^{26}$ we rewrite the free energy of Flory and Rehner ${ }^{20}$ in the form

$$
\begin{aligned}
\hat{W}(\mathbf{F}, \mu)= & \frac{1}{2} N k T\left[F_{i K} F_{i K}-3-2 \log (\operatorname{det} \mathbf{F})\right] \\
& -\frac{k T}{v}\left[(\operatorname{det} \mathbf{F}-1) \log \left(\frac{\operatorname{det} \mathbf{F}}{\operatorname{det} \mathbf{F}-1}\right)+\frac{\chi}{\operatorname{det} \mathbf{F}}\right] \\
& -\frac{\mu}{v}(\operatorname{det} \mathbf{F}-1),
\end{aligned}
$$

where $N$ is the number of polymer chains in the gel divided by the volume of the gel in the reference state, $k T$ is the temperature in the unit of energy, $v$ is the volume per solvent molecule, and $\chi$ is a dimensionless parameter characterizing the enthalpy of mixing. In writing Eq. (9), the reference configuration is taken to be the dry network, and $\mu=0$ when the solvent is in the pure liquid state in equilibrium with its own vapor.

Assuming that the small molecules diffuse in the gel and that the coefficient of diffusion of the solvent molecules $D$ is isotropic and independent of deformation gradient and concentration, Hong et al. ${ }^{26}$ expressed the mobility tensor as

$$
M_{K L}=\frac{D}{v k T}(\operatorname{det} \mathbf{F}-1) H_{i K} H_{i L},
$$

where $H_{i K}$ is the transpose of the inverse of the deformation gradient, namely, $H_{i K} F_{i L}=\delta_{K L}$. In writing Eq. (10) the reference state is taken to be the dry network.

We normalize the free-energy density by $k T / v$, the stress by $k T / v$, and the chemical potential by $k T$. The theory has no intrinsic length scale or intrinsic time scale. Let $L$ be a characteristic length in a boundary-value problem. We normalize all the other lengths by $L$, and normalize the time by $L^{2} / D$.

A representative value of the volume per solvent molecule is $v=10^{-28} \mathrm{~m}^{3}$. At room temperature, $k T=4 \times 10^{-21} \mathrm{~J}$ and $k T / v=4 \times 10^{7} \mathrm{~Pa}$. The Flory-Rehner free-energy density function introduces two dimensionless material parameters: $N v$ and $\chi$. The dry network has a shear modulus $N k T$ 
under the small-strain conditions, with the representative values $N k T=10^{4}-10^{7} \mathrm{~N} / \mathrm{m}^{2}$, which gives the range $N v$ $=10^{-4}-10^{-1}$. The parameter $\chi$ is a dimensionless measure of the enthalpy of mixing, with representative values $\chi$ $=0-1.2$. For applications that prefer gels with large swelling ratios, materials with low values of $\chi$ are used. In the numerical examples below, we will take the values $N v=10^{-3}$ and $\chi=0.2$. The coefficient of diffusion for water is $D=8$ $\times 10^{-10} \mathrm{~m}^{2} / \mathrm{s}$.

\section{FINITE ELEMENT FORMULATION}

Subject to external forces and immersed in an environment, a gel will deform and exchange solvent molecules with the environment. If the applied forces are time-independent, and the chemical potential of the solvent in the environment is homogenous and time-independent, after some time the gel will reach a state of thermodynamic equilibrium, in which the chemical potential of the solvent inside the gel becomes homogeneous and takes the same value as that in the environment. The time needed for the gel to equilibrate scales with $L^{2} / D$, where the length $L$ is a characteristic length of the gel. When the gel equilibrates, both the deformation of the network and the concentration of the solvent in the gel can still be inhomogeneous, as studied by Zhao et $a l^{32}$ and Hong et al. ${ }^{29}$

In many applications, however, the gel may not be in a state of thermodynamic equilibrium, so that the chemical potential of the solvent in the gel is inhomogeneous. The field of chemical potential of the solvent $\mu(\mathbf{X}, t)$ and the field of displacement of the network $x_{i}(\mathbf{X}, t)$ evolve concurrently. To study this coevolution, we now use the above theory to formulate a finite element method.

Multiply Eq. (6) by a test function $\xi_{i}(\mathbf{X})$, integrate over the volume of the gel, and then apply the divergence theorem, we obtain that

$$
\int \frac{\partial \hat{W}}{\partial F_{i K}} \frac{\partial \xi_{i}}{\partial X_{K}} d V=\int B_{i} \xi_{i} d V+\int T_{i} \xi_{i} d A
$$

In deriving Eq. (11), we have used the condition of mechanical equilibrium on the surface, Eq. (7), and the equation of state, Eq. (5). The last integral in Eq. (11) extends to the part of the surface over which the traction $T_{i}$ is prescribed. On the remaining part of the surface, the position of the network must be prescribed, and the test function $\xi_{i}(\mathbf{X})$ is set to be zero. The conditions of mechanical equilibrium in Eqs. (5)-(7) are equivalent to a single statement: the gel is in mechanical equilibrium if the weak form (Eq. (11)) holds for any arbitrary test function $\xi_{i}(\mathbf{X})$. Indeed, this statement is a direct consequence of the conditions of local equilibrium, as discussed in Hong et al. ${ }^{26,29}$

Multiply Eq. (2) by another test function $\zeta(\mathbf{X})$, integrate over the volume of the gel, and then apply the divergence theorem, we obtain that

$$
\begin{aligned}
& \int\left(\frac{\partial^{2} \hat{W}}{\partial \mu \partial F_{j L}} \frac{\partial F_{j L}}{\partial t}+\frac{\partial^{2} \hat{W}}{\partial \mu^{2}} \frac{\partial \mu}{\partial t}\right) \zeta d V-\int M_{K L} \frac{\partial \mu}{\partial X_{L}} \frac{\partial \zeta}{\partial X_{K}} d V \\
& =-\int r \zeta d V-\int i \zeta d A .
\end{aligned}
$$

In deriving Eq. (12) we have replaced the concentration using the equation of state Eq. (4), and replaced the flux by using the kinetic Eq. (8). The last integral in Eq. (12) extends to the part of the surface over which the rate of injection $i$ is prescribed. On the remaining part of the surface, the chemical potential of the solvent must be prescribed, and the test function $\zeta(\mathbf{X})$ is set to be zero. Thus, the number of solvent molecules is conserved if the weak form [Eq. (12)] holds for arbitrary test function $\zeta(\mathbf{X})$.

We now discretize the governing Eqs. (11) and (12) in space. Interpolate the position vector $x_{i}(\mathbf{X}, t)$ and the chemical potential $\mu(\mathbf{X}, t)$ as

$$
\begin{aligned}
& x_{i}(\mathbf{X}, t)-X_{i}=N_{a}(\mathbf{X}) u_{a i}(t), \\
& \mu(\mathbf{X}, t)=N_{a}(\mathbf{X}) \mu_{a}(t) .
\end{aligned}
$$

The index $a$, as well as the index $b$ below, is reserved for nodes; repeated $a$ (or $b$ ) implies summation over all nodes in the body. The quantities $u_{a i}(t)$ and $\mu_{a}(t)$ are the displacement and chemical potential associated with node $a$. The shape functions $N_{a}(\mathbf{X})$ can be constructed in several ways; we adopt the eight-node brick elements. The same approach of discretization is applied to the test functions $\xi_{i}(\mathbf{X})$ and $\zeta(\mathbf{X})$.

Substituting Eqs. (13) and (14) into Eq. (11), and invoking the arbitrariness of the test function $\xi_{i}(\mathbf{X})$, we obtain that

$$
\int \frac{\partial \hat{W}}{\partial F_{i K}} \frac{\partial N_{a}}{\partial X_{K}} d V=\int B_{i} N_{a} d V+\int T_{i} N_{a} d A .
$$

Equation (15a) is valid at all times. Taking a derivative with respect to time, we obtain that

$$
\begin{aligned}
& \frac{d u_{b j}}{d t} \int \frac{\partial^{2} \hat{W}}{\partial F_{i K} \partial F_{j L}} \frac{\partial N_{a}}{\partial X_{K}} \frac{\partial N_{b}}{\partial X_{L}} d V \\
& +\frac{d \mu_{b}}{d t} \int \frac{\partial^{2} \hat{W}}{\partial F_{i K} \partial \mu} \frac{\partial N_{a}}{\partial X_{K}} N_{b} d V \\
& =\int \frac{d B_{i}}{d t} N_{a} d V+\int \frac{d T_{i}}{d t} N_{a} d A .
\end{aligned}
$$

Substituting Eqs. (13) and (14) into Eq. (12), and invoking the arbitrariness of the test function $\zeta(\mathbf{X})$, we obtain that

$$
\begin{aligned}
& \frac{d u_{b j}}{d t} \int \frac{\partial^{2} \hat{W}}{\partial \mu \partial F_{j L}} \frac{\partial N_{b}}{\partial X_{L}} N_{a} d V+\frac{d \mu_{b}}{d t} \int \frac{\partial^{2} \hat{W}}{\partial \mu^{2}} N_{b} N_{a} d V \\
& \quad-\mu_{b} \int M_{K L} \frac{\partial N_{b}}{\partial X_{L}} \frac{\partial N_{a}}{\partial X_{K}} d V \\
& =-\int r N_{a} d V-\int i N_{a} d A .
\end{aligned}
$$

Equations (15b) and (16) are ordinary differential equations that concurrently evolve the nodal values of the dis- 
placement and the chemical potential $u_{b j}(t)$ and $\mu_{b}(t)$. The ordinary differential equations can be rewritten in a matrix form

$$
\mathbf{R} \frac{d \boldsymbol{\phi}}{d t}+\mathbf{K} \boldsymbol{\phi}=\mathbf{P}
$$

The column $\boldsymbol{\phi}$ lists values of $u_{b j}(t)$ and $\mu_{b}(t)$ of all nodes. The column $\mathbf{P}$ collects terms on the right-hand side of Eqs. (15a) and (16). The matrix $\mathbf{R}$ collects the integrals in Eqs. (15) and (16) involving the second derivatives of the freeenergy function. The matrix $\mathbf{K}$ collects the integral in Eq. (16) involving the mobility tensor.

We next discretize the problem in time. It is straightforward to solve Eq. (17) by an explicit method. However, the stability condition for explicit scheme is very restrictive, especially when the mobility tensor varies with swelling. The peak in the mobility tensor will cut down the allowable time step. Therefore, we use an implicit method to discretize the equation in time. The increment in the generalized nodal variable $\Delta \boldsymbol{\phi}$ at current time $t+\Delta t$ is obtained by solving the following equation:

$$
\mathbf{A}^{t+\Delta t} \Delta \boldsymbol{\phi}=\mathbf{Y}^{t+\Delta t}
$$

with

$$
\begin{aligned}
\mathbf{A}^{t+\Delta t} & =\frac{\mathbf{R}^{t+\Delta t}}{\Delta t}+\mathbf{K}^{t+\Delta t}, \\
\mathbf{Y}^{t+\Delta t} & =\mathbf{P}^{t+\Delta t}-\mathbf{R}^{t} \frac{d \boldsymbol{\phi}^{t}}{d t}-\mathbf{K}^{t} \boldsymbol{\phi}^{t} .
\end{aligned}
$$

The superscript denotes the time of the iterative step.

There are number of ways to solve Eq. (18). One way is to use direct solver, which requires that the matrix $\mathbf{A}$ be positive definite. However, effective stiffness matrix $\mathbf{A}$ is indefinite in the present formulation because the positivedefinite mobility tensor $\mathbf{M}$ contributes to the negative of matrix A [Eq. (16)]. Moreover, at the critical point where material instability occurs, the effective stiffness matrix $\mathbf{A}$ is singular, which fails the standard iteration procedure, such as Newton-Raphson method. Explicitly, the solution quickly diverges and leads to unphysical predictions (e.g., unbounded displacement increment). Various methods have been developed to resolve this numerical difficulty, such as Riks method $^{33}$ that has been used in ABAQUS. To overcome the convergence difficulty, we adopt a simple method that can be easily implemented in a typical finite element code. We replace A by

$$
\mathbf{A}^{*}=\mathbf{A}+\alpha \mathbf{I},
$$

where $\mathbf{I}$ is the identity matrix and $\alpha$ is a positive number as a penalty to ensure the positive definiteness of $\mathbf{A}^{*}$. This modification eliminates the singularity of the effective stiffness matrix to ensure a convergent result is reached. This method has been extensively used in finite element analysis, especially in the solution of eigenvalues and eigenvectors of a singular stiffness matrix. ${ }^{34}$

It is important to note that the solution to Eq. (18) is independent of $\alpha$. This is because the convergence is char-

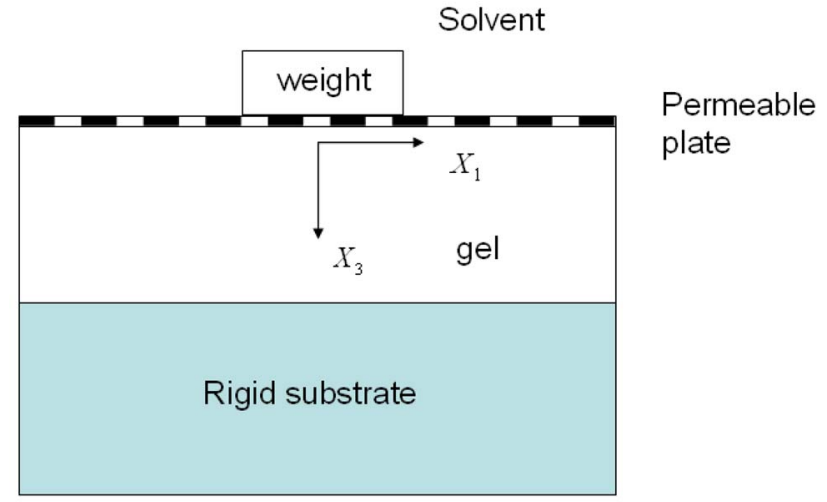

FIG. 3. (Color online) An illustration showing a fully swelling gel bonded to a right substrate and subject to an applied weight via a plate permeable to the small molecules.

acterized by $\mathbf{Y}=0$, or $\mathbf{R}^{t}\left(d \boldsymbol{\phi}^{t} / d t\right)+\mathbf{K}^{t} \boldsymbol{\phi}^{t}=\mathbf{P}^{t}$, whether we use A or $\mathbf{A}^{*}$. As to be shown in Section $\mathrm{V}$, the present method is able to study both the surface wrinkling featured with short wavelength instabilities and structure buckling featured with long wavelength instabilities.

Another way to solve Eq. (18) is to use an iterative solver, which does not require positive definiteness of matrix A. However, in numerical implementation, the iterative solver may "overshoot" the results for the current iteration step based on the previous iteration step, which might lead to nonphysical phenomena, for instance, $\operatorname{det} \mathbf{F}<1$. Conceptually, det $\mathbf{F}$ must be greater than one in this formulation to ensure the dry gel is incompressible. To resolve this nonphysical prediction, a very small incremental step should be used. Alternatively, the modification of the effective stiffness matrix $\mathbf{A}$ of Eq. (21) can be used for this purpose.

A common issue in modeling the swelling and shrinking of gels is the instability, which leads to zero eigenvalues of effective stiffness matrix A. To maintain the stability and convergence, the modification of the effective stiffness matrix A [Eq. (21)] is employed. This finite element formulation has been implemented in the ABAQUS/standard finite element program via its USER-ELEMENT subroutine, where the implicit time discretization and direct solver are employed.

\section{NUMERICAL EXAMPLES}

This section demonstrates the finite element method by analyzing several time-dependent processes of concurrent deformation and migration. Wherever possible, efforts are made to compare the numerical results with solutions obtained by using other methods, and with experimental observations.

\section{A. A gel drains under a weight}

The performance of the finite element method is tested by comparing the numerical results obtained by using the finite element method with those using a finite difference method for a problem studied in Ref. 26. Figure 3 illustrates a thin layer of a gel immersed in a pure liquid solvent. The gel first undergoes free swelling subject to no constraint and 
no applied forces. The swollen gel is then bonded to a rigid substrate, and subject to an applied weight. The solvent can migrate out from the top surface of the gel, and the gel thins down. The layer will eventually attain a new state of equilibrium.

Let $L$ be thickness of the dry network subject to no mechanical forces. This dry and undeformed configuration is used as the reference configuration, where a marker has the coordinates $X_{1}$ and $X_{2}$ in the plane of the layer, and the coordinate $X_{3}$ normal to the layer and pointing downwards. After free swelling and equilibrating with the pure liquid solvent, the layer swells by an isotropic stretch, $\lambda_{1}=\lambda_{2}=\lambda_{3}$ $=3.215$. The gel is then bonded to the rigid substrate, and subjected to a traction $s$, the weight divided by the area of the dry polymer. As the solvent migrates out, $\lambda_{1}$ and $\lambda_{2}$ remain unchanged, but $\lambda_{3}$ changes with time and position. The thickness of the gel is taken to be much smaller than the lateral dimensions of the gel, such that the field in gel is independent of $X_{1}$ and $X_{2}$. The functions $\lambda_{3}\left(X_{3}, t\right)$ and $\mu\left(X_{3}, t\right)$ are to be determined.

In a finite element model, we use twenty eight-node brick elements, stacked up one on top of another in the direction of the thickness. To model the full layer of the gel, we impose vanishing displacements and flux in lateral directions. The top surface of the gel is prescribed with the traction $s$ and the vanishing chemical potential, while the bottom surface of the gel is prescribed with the vanishing displacement and flux. Let $t_{\min }$ be the smallest time over which the solution is of interest to us, the size of the elements $l_{e}$ must be chosen such that $l_{e}<\sqrt{D t_{\min }}$. Once $l_{e}$ is chosen, the time step $\Delta t$ must also be limited by $\Delta t>l_{e}^{2} / D$.

Figure 4 compares the functions $\lambda_{3}\left(X_{3}, t\right)$ and $\mu\left(X_{3}, t\right)$ obtained from the finite element method in this paper and that from a finite difference method by Hong et al. ${ }^{26}$ The agreement is good. At the short-time limit, the weight is applied, but the solvent has no time to migrate out, so that the stretch is unchanged, $\lambda_{3}\left(X_{3}, 0\right)=3.215$, but the chemical potential jumps to a value higher than that of the external solvent, $\mu\left(X_{3}, 0\right)>0$. At the long-time limit, the chemical potential in the gel equilibrates with that of the solvent, $\mu\left(X_{3}, 0\right)=0$, and the stretch reduces to a new value. As a consequence of the conditions of local equilibrium, the top surface of the gel $\left(X_{3}=0\right)$ reaches the long-time limit instantaneously, with the vanishing chemical potential as fixed by the external solvent, and the low stretch. In a short time, the interior of the gel is still largely in the state of short-time limit. As the time processes, the solvent migrates out gradually, and the entire gel evolves toward the long-time limit.

\section{B. Free swelling of a cubic gel}

The free swelling of a cube of a gel, side $L$ in the dry state is studied. Conditions of symmetry are imposed, so that only one-eighth of the cube is modeled, using 512 brick elements. Figure 5 shows the distribution of dimensionless true stress $v \sigma_{x x} / k T$ on swelling configurations (keeping the relative ratio of volumes) at different time, characterized by nondimensional parameter $D t / L^{2}$. The true stress $\sigma_{i j}$ relates to the nominal stress $s_{i K}$ by
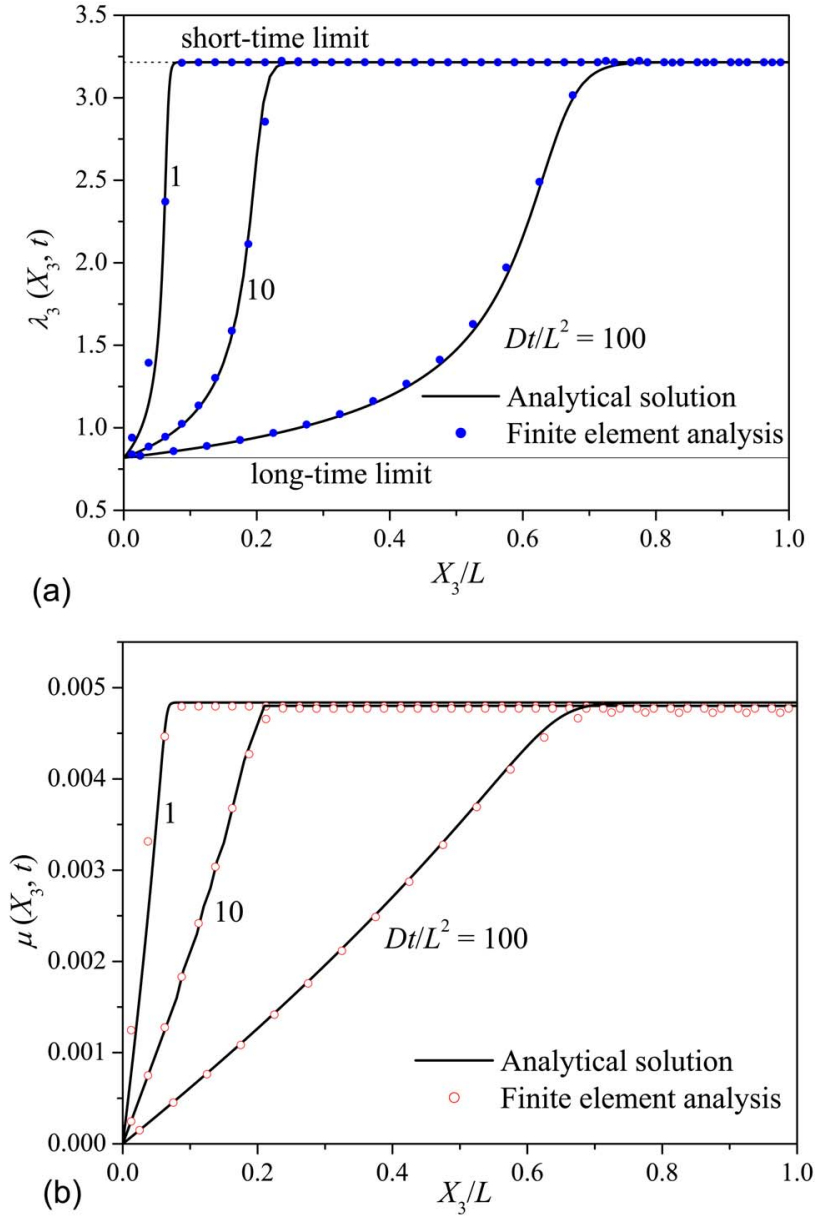

FIG. 4. (Color online) A gel $\left(N v=10^{-3}, \quad \chi=0.2\right)$ is subject to a nominal stress $v s / k T=-0.05$. The finite element results are marked by discrete dots, compared with the analytical solution marked by solid lines. The stretch $\lambda_{3}$ (a) and chemical potential $\mu$ (b) are inhomogeneous and evolves from the short-time limit to the long-time limit, which shows the creep behavior of a gel subject to mechanical loading.

$$
\sigma_{i j}=\frac{s_{i K} F_{j K}}{\operatorname{det} \mathbf{F}}
$$

Immediately upon the beginning of the swelling, the homogeneous gel [Fig. 5(a)] swells inhomogeneously [Fig. 5(b) for $\left.D t / L^{2}=1.25\right]$. The corners that have the largest contact surface with the solvent swell first, followed by the edges and then other parts of the gel, which leads to a bowl-like surface of the gel and generates compressive stress to the gel, similar to beam bending. As time goes on [Fig. 5(c) for $\left.D t / L^{2}=25\right]$, the swelling at the edges and the centers catch up with the swelling at the corners, so that the bowl-like surfaces become less concave. However, the deformation of the swelling gel is still inhomogeneous. This bowl-like surface in swelling gels has been observed experimentally that the gels swell faster at the corners than at the sides and the centers. ${ }^{35}$ It is also noticed that the compressive stress decreases during swelling. As the swelling process reaches equilibrium [Fig. 5(d) for $D t / L^{2} \rightarrow \infty$ ], the fully swelling gel recovers the shape identical to the original one and becomes totally homogeneous with vanishing stress and large volumetric change. 


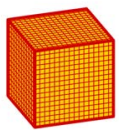

(a) $D t / L^{2}=0$

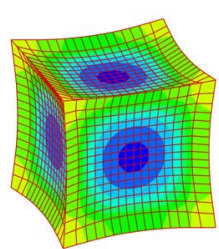

(b) $D t / L^{2}=1.25$
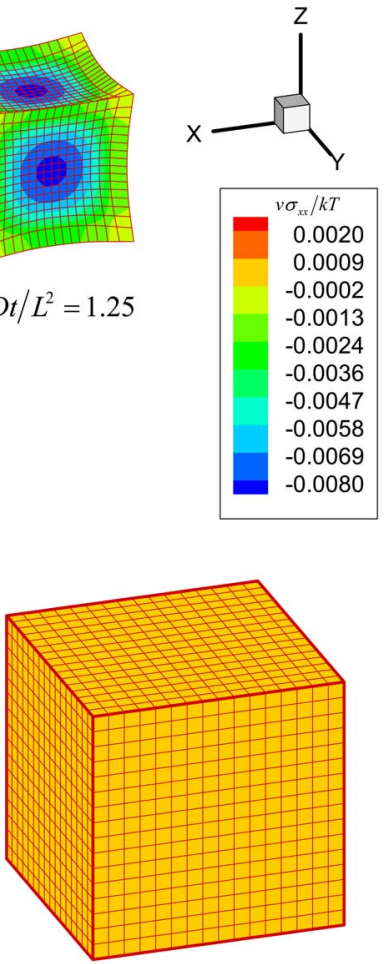

(d) $D t / L^{2} \rightarrow \infty$ (c) $D t / L^{2}=25$

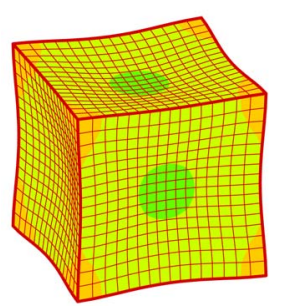

FIG. 5. (Color online) Contour plots of the nondimensional Cauchy stress $v \sigma_{x x} / k T$ for a free swelling cubic gel (size $L \times L \times L$ ) at different time scale characterized by nondimensional parameter $D t / L^{2}$. The initial state (i.e., dry gel for $D t / L^{2}=0$ ) is shown in (a). During the free swelling, nonuniform deformations appear for (b) and (c) with $D t / L^{2}=1.25$ and $D t / L^{2}=25$, respectively. Compressive stresses are also developed during free swelling. At the final state (or equilibrium state with $D t / L^{2} \rightarrow \infty$ ), the fully swelling gel recovers the shape identical to the original one and becomes totally homogeneous with vanishing stress and large volumetric change. The same scale is used to show the large volumetric change.

\section{Free swelling of a thin sheet}

The compressive stress generated due to free swelling may lead to buckling of gels that have been extensively observed in experiments (e.g., Ref. 36). This paper studies the buckling of a free swelling thin film gel. The aspect ratio of the in-plane dimension and the thickness is 12. Figure 6 shows the generation of wrinkling during swelling [Fig. 6(b)] starting from a homogeneous gel [Fig. 6(a)]. The similar patterns of crease were observed experimentally by Tanaka et $a l .{ }^{13}$ Besides the gels with geometric features (e.g., thin film), the gels have geometric imperfections that exist for almost all experiments also have buckling patterns during free swelling, such as in Sayil and Okay's ${ }^{36}$ experiments.

The wrinkles generated during free swelling may be troublesome in applications. Experimentally, the swelling ratio of gels, one of the most important parameters to characterize gels, is usually measured gravimetrically as a function of time. In other words, one usually measures the weight change as a function of time during swelling. The gained weight of a gel is proportional to the total number of the small molecules $v C$ absorbed by the swelling gel. To study whether the wrinkles really affect the weight change, we compare the finite element results having wrinkles [Figs. $6(b)]$ with the numerical solution restraining the wrinkles during free swelling.

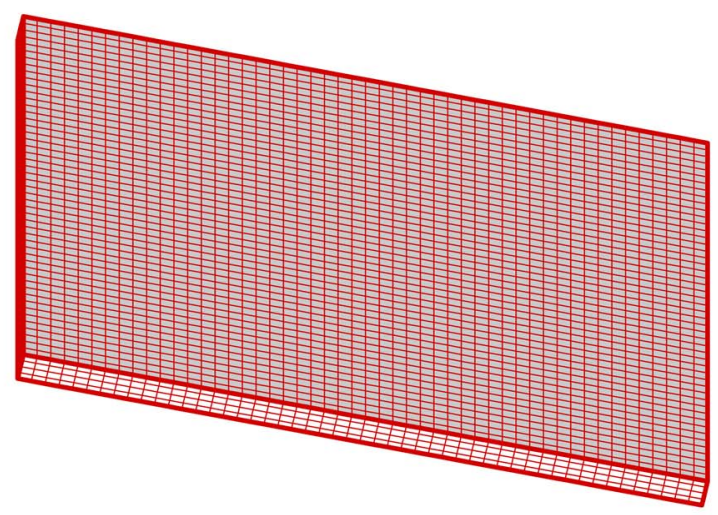

(a)

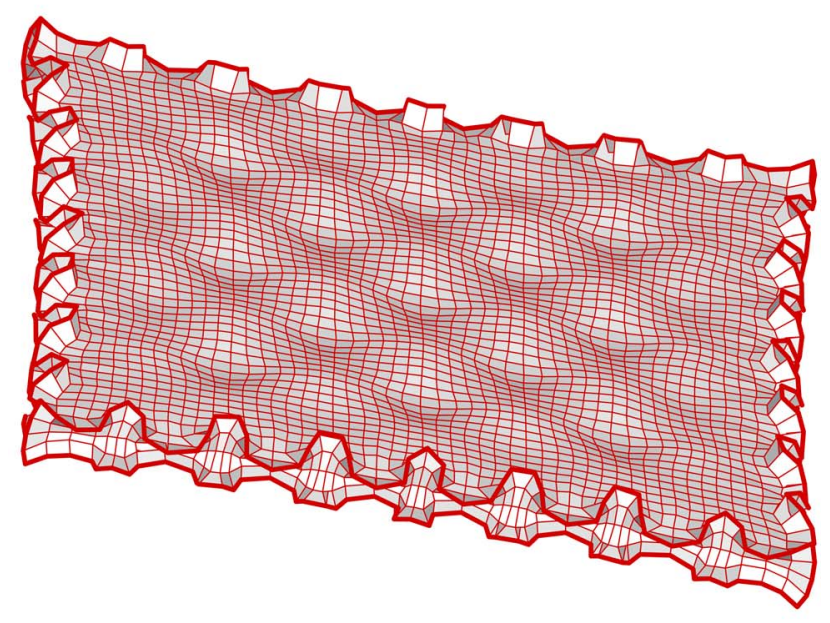

(b)

FIG. 6. (Color online) Shapes of (a) a dry gel $\left(D t / L^{2}=0\right)$ and a swelling gel $\left(D t / L^{2}=7.5\right)$. During the free swelling, the wrinkle patterns are developed. The scales for (a) and (b) are different.

Figure 7 shows the total number of the small molecules $v C$ inside the swelling gel as a function of time. The finite element results are marked by discrete dots and the numerical solution is given by a solid line. The finite element results fairly agree with the numerical results, although there are some discrepancies, which could be attributed to the different aspect ratios used, namely, 12 for finite element results and infinite for numerical results. This study concludes that the wrinkles do not significantly affect the weight change as a function of time. Therefore, people can ignore the wrinkles if they only concern the weight change.

\section{Swelling of a partially constrained gel}

A soft gel bonded to a stiff gel provides a model system to study pattern formation in elastic bodies. The kinetics of swelling soft gels on stiff gels is very important, especially in biological problems, such as the development of embryos. ${ }^{37}$ In this section, we will study the kinetics of swelling of soft gels bonded to nonswelling stiff gels in striped and circular geometries. This system was experimentally studied and an equilibrium state was determined theoretically. ${ }^{18}$

Figure 8(a) shows the strip geometry consisting of one thin strip of soft gel bonded to another thin strip of stiff gel. 


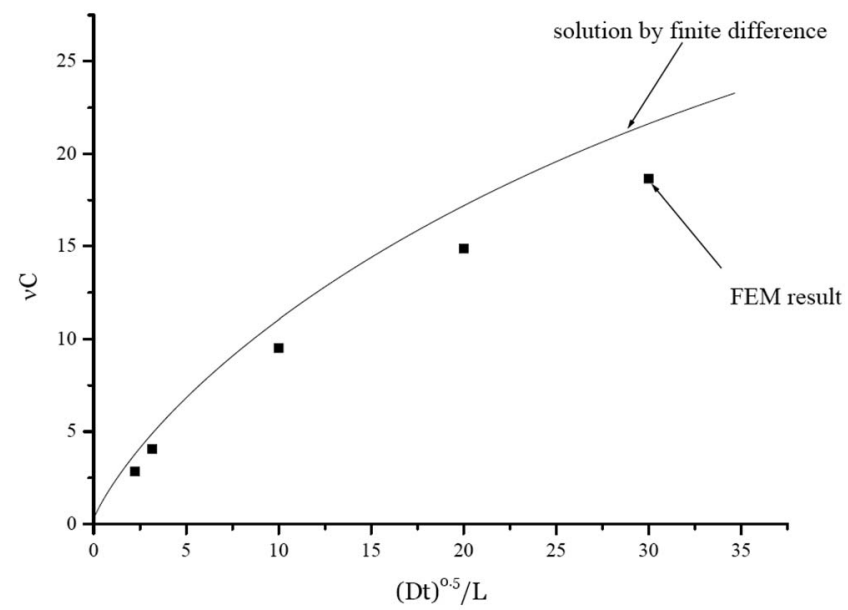

FIG. 7. The total mass of the small water molecules per unit volume of the dry gel as a function of time during free swelling of a thin film gel. The finite element results are given by the discrete dots and the numerical solution is marked by the solid line. It shows that the buckling pattern does not significantly affect the total mass of the small water molecules inside the free swelling thin film gels that are important in many applications.

Let $h$ and $w$ denote the thickness and width of the soft gel, respectively. The soft gel is subjected to vanishing displacements and flux at the interface between soft and stiff gels. The zero chemical potential is enforced at the gel/solvent interface. Figures 8(b) and 8(c) show the buckling patterns with the same scale at $D t / h^{2}=1.4$ and $D t / h^{2} \rightarrow \infty$ (long-time limitation or equilibrium state). It is observed that the transient problem has distinct buckling pattern compared with the equilibrium state. The buckling results from the fact that fast swelling at the free edges (e.g., top of the soft gel) generates compressive stress at the soft/stiff gels interfaces, as clearly shown in the contour plot of nondimensional stress $v \sigma_{x x} / k T$, which is also similar to beam bending. The wavelength $\lambda$ of the equilibrium state is consistent with Mora and Boudaoud's ${ }^{18}$ theoretical analysis, i.e., $\lambda=3.256 h$.

Figure 9(a) shows the geometry in which a disk of a soft gel is bonded to a stiff gel with the same shape, where $R_{i}$ and $R_{o}$ are inner and outer radius, respectively. We take $R_{i}$ $=3.5 \mathrm{~mm}$ and $R_{o}=5 \mathrm{~mm}$ in this study. Vanishing displacement and flux boundary conditions are imposed at the inner radius and the vanishing chemical potential is used at the outer radius. Figures 9(b) and 9(c) show the buckling patterns at $D t /\left(R_{o}-R_{i}\right)^{2}=4.45$ and $D t /\left(R_{o}-R_{i}\right)^{2} \rightarrow \infty$ (i.e., equilibrium state). A coronal buckling pattern [Fig. 9(b)] observed during the swelling finally disappears at the equilibrium state [Fig. 9(c)].

The different swelling behaviors of a soft, strip gel and a soft, disk gel indicate that the geometric shapes of the gels play a very important role on the kinetics of swelling of gels. These differences (Figs. 8 and 9) can be qualitatively understood in the following. The swelling of the strip gel bonded to a stiff gel has an analogy to beam bending, i.e., compressive stress at the interface between soft and stiff gels. Therefore, no matter the thickness of the soft gel, the swellinginduced compressive stress leads to wrinkles, even at the equilibrium state as shown in Fig. 8(c). However, the analogy to beam bending is not valid for the disk gel due to the geometric constrain in the circumferential direction. In fact, it is noticed that the wrinkles for the disk gels [Fig. 9(b)] are very similar to that of the free swelling thin film gels [Fig. 6(b)], not buckling of strip gels [Figs. 8(b) and 8(c)]. Therefore, at the equilibrium state [Fig. 9(c)], the wrinkling disappears and the fully swelling state is similar to that of the free swelling thin film gels.

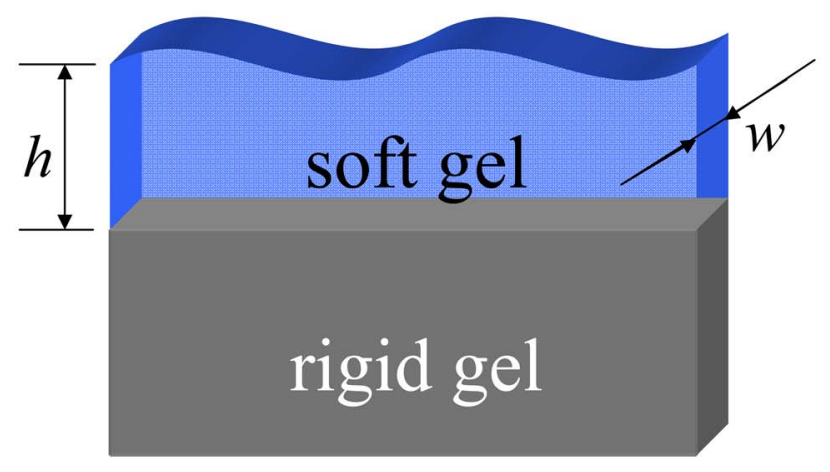

(a)

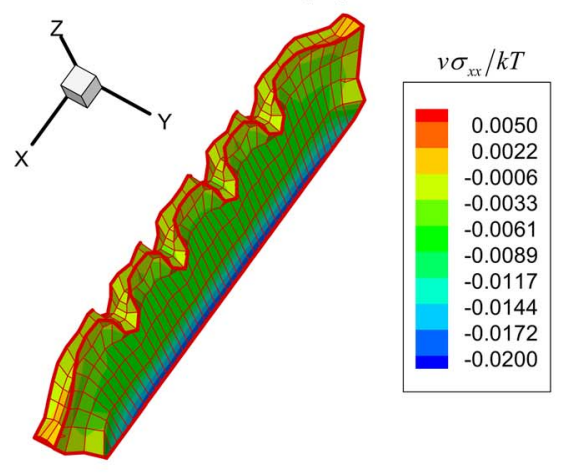

(b)

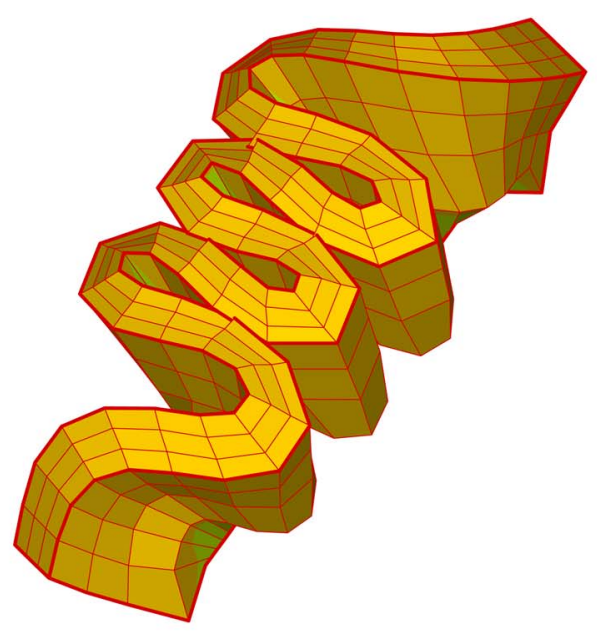

(c)

FIG. 8. (Color online) A swelling soft, strip gel bonded to a nonswelling stiff, strip gel. An illustration of the system of a soft gel and a stiff gel (a). During swelling $\left(D t / L^{2}=1.4\right)$, the soft gel wrinkles due to the compressive stress (b). The wrinkles grow and remain at the equilibrium state $\left(D t / L^{2}\right.$ 


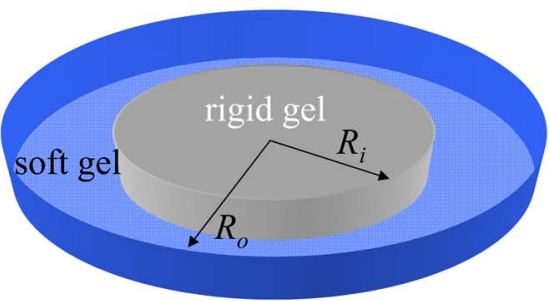

(a)
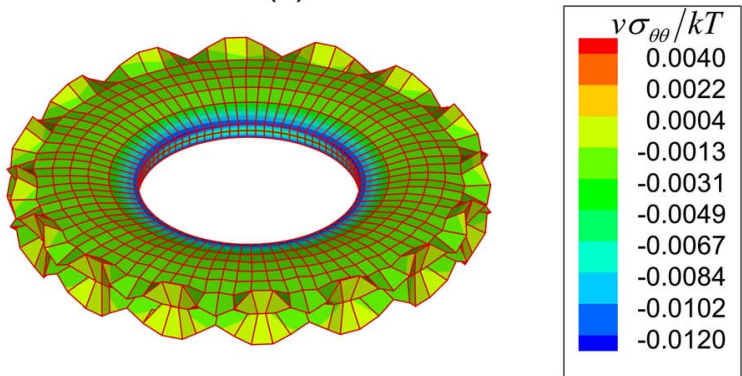

(b)

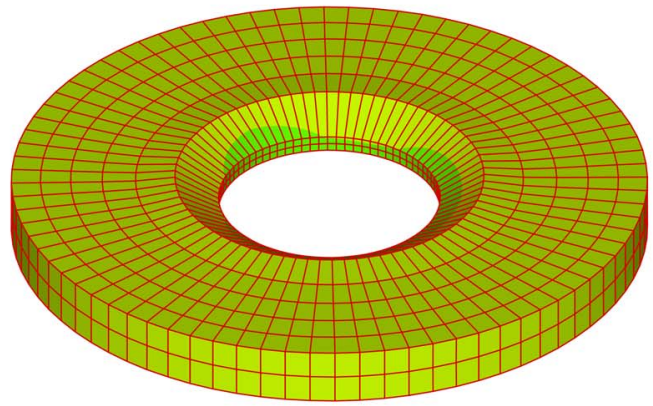

(c)

FIG. 9. (Color online) A swelling soft, disk gel bonded to a nonswelling stiff, disk gel. An illustration of the system of a soft gel and a stiff gel (a). During swelling $\left(D t / L^{2}=4.45\right)$, the soft gel generates coronal wrinkles due to the compressive stress (b). At the equilibrium state $\left(D t / L^{2} \rightarrow \infty\right)$ the coronal wrinkles disappear and the fully swelling gel recovers the shape similar to the original one.

\section{SUMMARY}

We have developed a finite element method to simulate concurrent large deformation and mass transport for gels. The method is implemented in ABAQUS/standard finite element program via its USER-ELEMENT subroutine. Numerical examples showed that this program is accurate by comparing with the available numerical solutions. The program has been used to study several time-dependent processes of swelling gels, such as draining of fully swollen gels due to weight, free swelling-induced surface instability, and buckling pattern formation due to partially confined swelling. Because the potential sensitivity of material models (i.e., freeenergy density and mobility tensor) on kinetic behaviors of gels, we plan to study this effect in the future work.

\section{ACKNOWLEDGMENTS}

The work at ASU is supported by a startup grant for $\mathrm{HJ}$ from the Fulton School of Engineering and partially by the NSF through Grant No. CMMI-0700440. The work at Harvard is supported by the DARPA through a project on programmable matter, and by the NSF through a project on Large Deformation and Instability in Soft Active Materials.

${ }^{1}$ W. Pilnik and F. M. Rombouts, Carbohydr. Res. 142, 93 (1985).

${ }^{2}$ F. Fischelghodsian, L. Brown, E. Mathiowitz, D. Brandenburg, and R. Langer, Proc. Natl. Acad. Sci. U.S.A. 85, 2403 (1988).

${ }^{3}$ R. Duncan, Nat. Rev. Drug Discovery 2, 347 (2003).

${ }^{4}$ B. Jeong, Y. H. Bae, D. S. Lee, and S. W. Kim, Nature (London) 388, 860 (1997).

${ }^{5}$ R. Langer, Nature (London) 392, 5 (1998).

${ }^{6}$ Y. Luo and M. S. Shoichet, Nature Mater. 3, 249 (2004).

${ }^{7}$ A. P. Nowak, V. Breedveld, L. Pakstis, B. Ozbas, D. J. Pine, D. Pochan, and T. J. Deming, Nature (London) 417, 424 (2002).

${ }^{8}$ D. J. Beebe, J. S. Moore, J. M. Bauer, Q. Yu, R. H. Liu, C. Devadoss, and B. H. Jo, Nature (London) 404, 588 (2000).

${ }^{9}$ L. Dong, A. K. Agarwal, D. J. Beebe, and H. R. Jiang, Nature (London) 442, 551 (2006).

${ }^{10}$ A. Sidorenko, T. Krupenkin, A. Taylor, P. Fratzl, and J. Aizenberg, Science 315, 487 (2007).

${ }^{11}$ M. Kleverlaan, R. H. van Noort, and I. Jones, Proceedings of the SPE/ IADC Conference, Amsterdam, Netherlands, 2005 (unpublished).

${ }^{12}$ K. Y. Lee, M. C. Peters, K. W. Anderson, and D. J. Mooney, Nature (London) 408, 998 (2000).

${ }^{13}$ T. Tanaka, S. T. Sun, Y. Hirokawa, S. Katayama, J. Kucera, Y. Hirose, and T. Amiya, Nature (London) 325, 796 (1987).

${ }^{14}$ V. Trujillo, J. Kim, and R. C. Hayward, Soft Matter 4, 564 (2008).

${ }^{15}$ T. Tanaka and D. J. Fillmore, J. Chem. Phys. 70, 1214 (1979).

${ }^{16}$ I. J. Suarez, A. Fernandez-Nieves, and M. Marquez, J. Phys. Chem. B 110, 25729 (2006).

${ }^{17}$ Y. Li and T. Tanaka, J. Chem. Phys. 92, 1365 (1990).

${ }^{18}$ T. Mora and A. Boudaoud, Eur. Phys. J. E 20, 119 (2006).

${ }^{19}$ H. Murata, B. J. Chang, O. Prucker, M. Dahm, and J. Ruhe, Surf. Sci. 570, 111 (2004).

${ }^{20}$ P. J. Flory and J. Rehner, J. Chem. Phys. 11, 521 (1943).

${ }^{21}$ C. J. Durning and K. N. Morman, J. Chem. Phys. 98, 4275 (1993).

${ }^{22}$ J. Dolbow, E. Fried, and H. D. Jia, J. Mech. Phys. Solids 52, 51 (2004).

${ }^{23}$ H. Tsai, T. J. Pence, and E. Kirkinis, J. Elast. 75, 69 (2004).

${ }^{24} \mathrm{H}$. Li, R. Luo, E. Birgersson, and K. Y. Lam, J. Appl. Phys. 101, 7 (2007).

${ }^{25}$ K. Sekimoto, J. Phys. II 1, 19 (1991).

${ }^{26}$ W. Hong, X. H. Zhao, J. X. Zhou, and Z. G. Suo, J. Mech. Phys. Solids 56, 1779 (2008).

${ }^{27}$ R. M. Bowen, Continuum Physics (Academic, New York, 1975).

${ }^{28}$ K. K. Westbrook and H. J. Qi, J. Intell. Mater. Syst. Struct. 19, 597 (2008).

${ }^{29}$ W. Hong, Z. Liu, and Z. Suo (submitted).

${ }^{30}$ N. Suematsu, K. Sekimoto, and K. Kawasaki, Phys. Rev. A 41, 5751 (1990).

${ }^{31}$ E. Birgersson, H. Lib, and S. Wua, J. Mech. Phys. Solids 56, 444 (2008).

${ }^{32}$ X. H. Zhao, W. Hong, and Z. G. Suo, Appl. Phys. Lett. 92, 3 (2008).

${ }^{33}$ E. Riks, ASME Trans. J. Appl. Mech. 39, 1060 (1972).

${ }^{34}$ K. J. Bathe, Finite Element Procedures in Engineering Analysis (PrenticeHall, Englewood Cliffs, NJ, 1982).

${ }^{35}$ E. C. Achilleos, R. K. Prud'homme, K. N. Christodoulou, K. R. Gee, and I. G. Kevrekidis, Chem. Eng. Sci. 55, 3335 (2000).

${ }^{36}$ C. Sayil and O. Okay, Polymer 42, 7639 (2001).

${ }^{37}$ E. Brouzes and E. Farge, Curr. Opin. Genet. Dev. 14, 367 (2004). 